\title{
Effects of Repetitive Transcranial Magnetic Stimulation Over Trunk Motor Spot on Balance Function in Stroke Patients
}

\author{
Cheol-Min Choi, MD, Jin-Hong Kim, MD, June-Kyung Lee, MD, Bong-Yeon Lee, MD, \\ Hoi-Sung Kee, MD, Kwang-Ik Jung, MD, Seo-Ra Yoon, MD
}

Department of Rehabilitation Medicine, Gwangju Veterans Hospital, Gwangju, Korea

\begin{abstract}
Objective To assess the efficacy of high-frequency repetitive transcranial magnetic stimulation (rTMS) on balance function in patients with chronic stroke.

Methods Thirty participants with chronic stroke were enrolled in this study. High frequency $(10 \mathrm{~Hz}) \mathrm{rTMS}$ was delivered with butterfly-coil on trunk motor spot. Each patient received both real and sham rTMS in a random sequence. The rTMS cycles (real or sham) were composed of 10 sessions each, administered over two weeks, and separated by a 4 -week washout period. Balance function was measured by Berg Balance Scale and computerized dynamic posturography to determine the effect of rTMS before and one day after the end of each treatment period, as well as at a 1-month follow-up.

Results The balance function was significantly improved after high frequency rTMS as compared with that after sham rTMS $(\mathrm{p}<0.05)$. There was no serious adverse effect in patients during the treatment period.

Conclusion In the chronic stroke patients, high frequency rTMS to the trunk motor area seems to be a helpful way to improve balance function without any specific adverse effects. Further studies are needed to identify the underlying mechanism and generate a detailed protocol.
\end{abstract}

Keywords Transcranial magnetic stimulation, Stroke, Postural balance, Hemiplegia

\section{INTRODUCTION}

Stroke is one of the reasons for death and disability in adults $[1,2]$. Balance deficits are frequently seen in stroke patients $[3,4]$ and associated with gait dysfunction, disability to perform independent daily living, and

Received January 18, 2016; Accepted April 27, 2016

Corresponding author: Seo-Ra Yoon

Department of Rehabilitation Medicine, Gwangju Veterans Hospital, 99, Cheomdanwolbong-ro, Gwangsan-gu, Gwangju 62284, Korea. Tel: +82-62602-6295, Fax: +82-62-602-6990, E-mail: standupmd@hanmail.net

ORCID: Cheol-Min Choi (http://orcid.org/0000-0003-3993-8437); Jin-Hong Kim (http://orcid.org/0000-0003-1871-8400); June-Kyung Lee (http:// orcid.org/0000-0002-9900-0961); Bong-Yeon Lee (http://orcid.org/0000-0002-2975-6333); Hoi-Sung Kee (http://orcid.org/0000-0002-7683-8366); Kwang-Ik Jung (http://orcid.org/0000-0002-9754-5925); Seo-Ra Yoon (http://orcid.org/0000-0002-3694-0710).

(c) This is an open-access article distributed under the terms of the Creative Commons Attribution Non-Commercial License (http://creativecommons.org/ licenses/by-nc/4.0) which permits unrestricted noncommercial use, distribution, and reproduction in any medium, provided the original work is properly cited. Copyright (C) 2016 by Korean Academy of Rehabilitation Medicine 
increased risk of falling $[5,6]$. As falling accidents can lead to severe outcomes such as bone fracture and traumatic brain injury, restoring the balance function is one of the important goals of rehabilitation [3,7-9].

Recently, use of transcranial magnetic stimulation (TMS) has received immense attention in stroke rehabilitation. Noninvasive brain stimulation techniques such as repetitive transcranial magnetic stimulation (rTMS) and transcranial direct current stimulation (tDCS) have been investigated as therapeutic interventions for various neurological disorders like motor deficits, aphasia and hemispatial neglect after stroke [10-14]. However, until date, there exists no study investigating the effects of rTMS on balance function in chronic stroke patients. Only, one study by Sohn et al. [15] demonstrates improvement in postural stability and lower extremity strength upon application of tDCS to hemiplegic stroke patients. The trunk muscles play an important role in postural balance and have a significant effect on the recovery of balance in case of stroke patients $[16,17]$.

Therefore, this study aims to investigate therapeutic effect of application of high frequency rTMS on balance function in patients with chronic stroke. We assumed that restoration of balance function is expedited by performing rTMS on trunk motor spot, and this operation is safe. In this regards, the study was performed with an aim to establish the clinical knowledge, which can be applied to chronic stroke patients with balance impairment through research of rTMS treatment paradigm.

\section{MATERIALS AND METHODS}

\section{Subjects}

Forty patients with balance impairment after stroke were enrolled in this study and 33 patients met the inclusion criteria. The inclusion criteria were as follows: (1) brain lesion detectable by magnetic resonance imaging or computed tomography which were taken at the onset of the symptom; (2) the location of subcortical lesion was in middle cerebral artery (MCA) territory; (3) the time gap between enrollment in the present study and occurrence of cerebral infarct was a minimum of 6 months and a maximum of 10 years; (4) mild to moderate balance impairment (score of Berg Balance Scale [BBS] was $\geq 20$ and $\leq 46$; and (5) Korean version of Mini-Mental State Examination score was $\geq 24$, indicative of cognitive ability, which was sufficient to understand the nature of study. The exclusion criteria were as follows: (1) having other neurologic problems, which can affect balance ability; (2) intake of drugs that can affect balance function; and (3) presence of contraindications for rTMS (pacemaker, cochlear implants, metal in the brain or skull, or history of epilepsy).

The ethics committee of Gwangju Veterans Hospital approved the study protocol, and written informed consent was obtained from all patients before the initiation of study.

\section{Study design}

Cross-over study design was used, and thereby all subjects participated in the experiments with both real stimulation and sham stimulation. The subjects were randomized by other healthcare professional who did not participate in this study, and were randomly divided into two groups considering their treatment order (real-sham or sham-real). There was an interval of 2 weeks between the treatment cycles to avoid interference of previous stimulation on next stimulation. Therefore, one group received the real-sham and another, the sham-real treatment sequence. Each treatment cycle lasted 2 weeks for 10 high-frequency rTMS sessions (Fig. 1).

\section{Intervention}

The figure-eight coil (MCF-B70; MagVenture, Farum, Denmark) and a Magstim Rapid stimulator (Magventure) were used as a real rTMS. Before rTMS, resting motor threshold (RMT) was measured after positioning the figure-eight coil over the cortex on the optimal location for obtaining trunk motor response (contralateral 9th thoracic erector spinae muscle). RMT was defined as a minimum intensity to induce motor evoked potential (MEP) $>50$ peak-to-peak amplitude in 5 of 10 consecutive trials on the contralateral 9th thoracic erector spinae muscles using needle electrode. If the motor response did not showed up at maximal stimulation, a symmetric position (mirror region) of the contralateral motor hotspot was decided as an alternative motor hotspot.

The patients were seated in a comfortable chair, and were asked to relax with their arms placed on the armrest in a comfortable position. Moreover, they stayed awake during the intervention. The stimulation coil was tangentially positioned over the motor cortical area of the 9th 


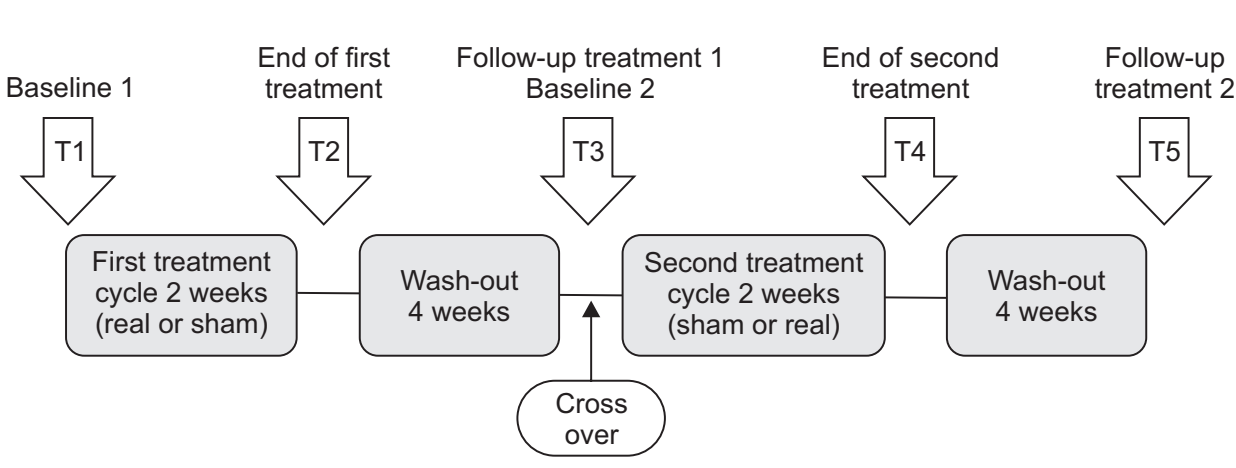

Fig. 1. Experimental design. For both groups, 10 real rTMS treatment and 10 sham rTMS treatment were performed within 2 weeks, separated by 4 weeks wash out period. $\mathrm{T}$, time of evaluation; rTMS, repetitive transcranial magnetic stimulation. thoracic erector spinae muscles. Real rTMS was delivered at $10 \mathrm{~Hz}$ and $90 \%$ of RMT for 5 seconds with 25 -second inter-train interval. A total of 1,000 pulses were delivered over a period of 10 minutes. For sham rTMS, the treatment cycles were same as that of real rTMS; however, sham coil (MCF-P-B70, MagVenture) which provides sound and the sensation of scalp similar to the real rTMS coil, but does not induce a magnetic field was used. In addition, the patients were not allowed to recognize whether it is the sham rTMS or not.

\section{Safety}

Occurrence of any possible adverse events in the patients was monitored by medical and neurological examination throughout the study. Vital signs, including blood pressure, heart rate, respiratory rate, and level of consciousness were checked before and after rTMS. During rTMS, a healthcare professional and a caregiver continuously monitored adverse events such as seizure.

\section{Balance test}

Quantified balance assessment was performed using computerized dynamic posturography, SMART Balance Master system (NeuroCom Inc., Clackamas, OR, USA). The SMART Balance Master system consists of three parts: two force platforms, a screen, and a main body of the computer. The patients were made to take off their shoes and stand in the middle of the force platform. Subsequently, they were asked to stare at the screen in front of them. On the day before the examination, the assessment method was fully explained to the patients, and the sensory organization test (SOT) for sensory limitation assessment and the rhythmic weight shift test for motor limitation assessment were performed.
Table 1. Six conditions of sensory organization test

\begin{tabular}{lcll}
\hline & Force platform & Eyes & Screen \\
\hline Condition 1 & Fixed & Open & Fixed \\
\hline Condition 2 & Fixed & Closed & Fixed \\
Condition 3 & Fixed & Open & Movement \\
Condition 4 & Sway & Open & Fixed \\
Condition 5 & Sway & Closed & Fixed \\
Condition 6 & Sway & Open & Movement \\
\hline
\end{tabular}

\section{Sensory limitation assessment}

SOT was performed for the assessment of sensory limitation. SOT represents the postural ability of the patients as an altering sway value when the patients were made to stay in a standing position under six conditions for 20 seconds (Table 1). After measurement of each condition thrice, the average of SOT was recorded and the measured sway was expressed as an equilibrium score.

\section{Motor limitation assessment}

In this study, rhythmic weight test was performed for the motor limitation assessment. The rhythmic weight test quantifies the patient's ability to move their center of gravity from forward to backward and left to right. It measures on-axis velocity $(\%)$ of the center of gravity according to the moving velocity (fast \& slow) and directional control (\%). In the present case, it was measured as one-axis movement of the body centerline per 3 seconds (3-second transitions) was regarded as low velocity and a one-axis movement of the body centerline per second (1-second transition) was regarded as a high velocity. On-axis velocity is dynamic weight movement in the intended direction and is expressed as an angular velocity per second. Directional control (DCL) is a variation of movement in the intended direction and is expressed as percentage (\%). They were measured thrice and averaged 
in a manner similar to sensory limitation assessment.

\section{Outcome measures}

The same levels of conservative treatments (muscle strengthening exercise, range of motion training) were executed for the patients during the treatment. The balance function assessment was executed three times using the SMART Balance Master system: one day before each treatment cycle, one day after, and one month after the end of treatment. Moreover, BBS was measured at the same time.

\section{Statistical analysis}

All analyses were performed with the SPSS ver. 18.0 (SPSS Inc., Chicago, IL, USA). The normal distribution was proved by Shapiro-Wilks test, and after that parametric test was used. A significance level was set at $\mathrm{p}<0.05$ in all analysis. After real or sham treatment, clinical outcomes were measured over time and analyzed by oneway analysis of variance (ANOVA) for repetitive measures.

For comparing the difference of effect between the real and the sham treatment, the results, which were from the treatment right after the end of the study and the followup values, which were obtained one month later, were calculated as percentages.

The ANOVA for repeated measures was performed using the treatment (sham, real) and time (end of treatment, follow-up) as a factor. When any significant effect was observed, the post-hoc comparisons were performed with paired Student t-test.

\section{RESULTS}

\section{Patient characteristics}

From a total of 33 patients, 3 were excluded from the study due to the following reasons: occurrence of latest cerebral infarction in one patient, occurrence of benign paroxysmal positional vertigo in one patient, worsening of general condition in one patient. Thereby 30 patients underwent the complete study until the final stage.

Out of 30 patients, 27 were male, 3 were female, and the age at the time of hospitalization was 60-85 years. In 15 patients, right hemisphere was affected; whereas other 15 patients had a lesion in the left hemisphere. Demographic details of each patient are summarized in Table 2. Sex, age, height, affected side, duration of symptoms, stroke subtype, and BBS did not show any significant differences between the two groups, which had a different treatment sequence.

\section{Adverse effects}

None of the patient experienced serious adverse event such as seizure. In addition, vital signs, including blood pressure, respiratory rate did not show any significant changes during the study. Two patients complained of scalp dysesthesia but it was not severe to the extent to stop the study and the symptoms disappeared in a few days after the completion of study.

\section{Balance function}

There was no statistical difference in the baseline of clinical measurements of two groups which had a different treatment order $(p>0.05)$. After real treatment, the factor 'time' statistically affected SOT, DCL left-right, on-axis velocity front-back, on-axis velocity left-right $(\mathrm{p}<0.05)$ in a significant manner, but no statistically significant effect was observed on DCL front-back ( $p=0.079$ ). On the other hand, there was no significant difference in clinical measurements after sham treatment ( $p>0.05$ ). Post-hoc analysis showed significant improvement in

Table 2. Demographic characteristics of patients

\begin{tabular}{lccc}
\hline & $\begin{array}{c}\text { Real-sham } \\
\text { group } \\
(\mathbf{n = 1 5})\end{array}$ & $\begin{array}{c}\text { Sham-real } \\
\text { group } \\
(\mathbf{n = 1 5})\end{array}$ & p-value ${ }^{\text {a) }}$ \\
\hline $\begin{array}{c}\text { Gender } \\
\text { (male:female) }\end{array}$ & $14: 1$ & $13: 2$ & \\
\hline Age (yr) & $67.1 \pm 3.8$ & $68.7 \pm 5.2$ & 0.342 \\
\hline $\begin{array}{l}\text { Height (cm) } \\
\text { Hemiplegia } \\
\text { (right:left) }\end{array}$ & $165.4 \pm 5.9$ & $162.7 \pm 10.4$ & 0.382 \\
\hline $\begin{array}{c}\text { Duration (mo) } \\
\text { Stroke subtype }\end{array}$ & $49.6 \pm 28.3$ & $44.0 \pm 29.9$ & 0.603 \\
\hline (CI:ICH) & $11: 4$ & $9: 6$ & 0.439 \\
\hline K-MBI & $71.3 \pm 7.4$ & $71.2 \pm 7.5$ & 0.961 \\
\hline BBS & $34.1 \pm 6.7$ & $32.9 \pm 7.0$ & 0.637 \\
\hline
\end{tabular}

Values are presented as mean \pm standard deviation. $\mathrm{CI}$, cerebral infarction; ICH, intracerebral infarction; KMBI, Korean version of Modified Barthel Index; BBS, Berg Balance Scale.

${ }^{\text {a) }}$ Student t-test. 
SOT score by comparing between baseline and the 2 time point of after treatments (baseline vs. end of treatment, $\mathrm{p}=0.003$; baseline vs. follow-up, $\mathrm{p}<0.05$ ) (Fig. 2). On-axis velocity and DCL left-right also showed results with the same aspects and they sustained for a month at the time point of follow-up. In case of DCL left-right and BBS, improvement in the results at the end of treatment was observed compared with those of sham treatment, but this effect did not reach statistical significance $(\mathrm{p}=0.132$, $\mathrm{p}=0.087$ ). Moreover, the results of the follow-up showed statistically significant improvement (Table 3). The percentage increases in clinical measures ware significantly greater for real rTMS than sham rTMS ( $\mathrm{p}<0.05)$. Furthermore, progress in improvement was noted between the end of treatment and follow-up (Fig. 3).

\section{DISCUSSION}

This study aims to investigate the effect of rTMS on the balance function when rTMS is applied to trunk motor area of chronic stroke patients. The muscles of trunk play an important role for maintaining appropriate body's posture, hence these muscles are highly affected with restoration of functions for patients with neurological damage after brain injury $[16,17]$.

Numerous studies have been carried out with emphasis on improvement of balance function. Karatas et al. [18] reported that strength of trunk muscles is related to the

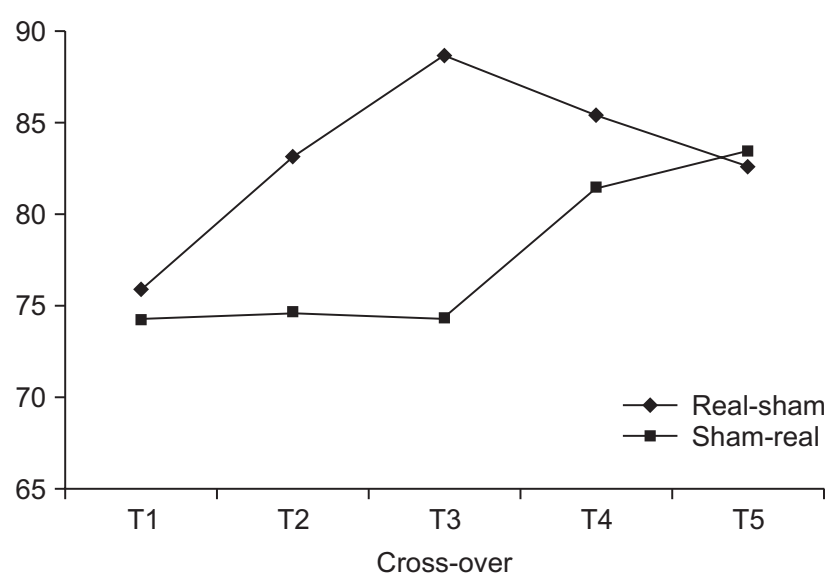

Fig. 2. Changes in sensory organization test (SOT) scores in accordance with treatment sequence (black diamonds, real-sham treatment; gray squares, sham-real treatment). In both groups, SOT score significantly improved after real treatment and improvement persisted for 1 month after treatment.

Table 3. Clinical evaluations according to treatment sequence $(n=30)$

\begin{tabular}{|c|c|c|c|c|c|c|}
\hline & \multirow[b]{2}{*}{ Treatment } & \multirow[b]{2}{*}{ Baseline } & \multirow[b]{2}{*}{ Post } & \multirow[b]{2}{*}{ Follow-up } & \multicolumn{2}{|c|}{ p-value } \\
\hline & & & & & $\begin{array}{l}\text { Post vs. } \\
\text { baseline }\end{array}$ & $\begin{array}{l}\text { Follow-up vs. } \\
\text { baseline }\end{array}$ \\
\hline \multirow[t]{2}{*}{ SOT } & Real rTMS & $75.1 \pm 8.8$ & $82.3 \pm 9.1$ & $86.1 \pm 9.3$ & $<0.05^{*}$ & $<0.05^{*}$ \\
\hline & Sham rTMS & $81.5 \pm 11.3$ & $80.1 \pm 10.2$ & $78.5 \pm 9.5$ & 0.616 & 0.265 \\
\hline \multirow[t]{2}{*}{ On-axis velocity left-right } & Real rTMS & $3.8 \pm 1.1$ & $4.8 \pm 1.2$ & $5.1 \pm 1.1$ & $<0.05^{*}$ & $<0.05^{*}$ \\
\hline & Sham rTMS & $4.6 \pm 1.3$ & $4.4 \pm 1.2$ & $4.3 \pm 1.2$ & 0.660 & 0.370 \\
\hline \multirow[t]{2}{*}{ On-axis velocity front-back } & Real rTMS & $2.3 \pm 0.8$ & $2.7 \pm 0.8$ & $3.0 \pm 0.8$ & $<0.05^{*}$ & $<0.05^{*}$ \\
\hline & Sham rTMS & $2.6 \pm 0.9$ & $2.9 \pm 0.9$ & $2.8 \pm 0.9$ & 0.312 & 0.384 \\
\hline \multirow[t]{2}{*}{ DCL left-right } & Real rTMS & $73.3 \pm 10.3$ & $78.7 \pm 9.1$ & $81.8 \pm 8.7$ & $<0.05^{*}$ & $<0.05^{*}$ \\
\hline & Sham rTMS & $77.8 \pm 10.4$ & $77.2 \pm 9.9$ & $76.4 \pm 9.7$ & 0.819 & 0.581 \\
\hline \multirow[t]{2}{*}{ DCL front-back } & Real rTMS & $47.0 \pm 18.3$ & $54.0 \pm 17.1$ & $58.5 \pm 17.4$ & 0.132 & $<0.05^{*}$ \\
\hline & Sham rTMS & $53.6 \pm 18.4$ & $52.2 \pm 17.8$ & $50.7 \pm 17.6$ & 0.776 & 0.534 \\
\hline \multirow[t]{2}{*}{ BBS } & Real rTMS & $33.5 \pm 6.8$ & $36.6 \pm 6.7$ & $37.9 \pm 6.4$ & 0.087 & $<0.05^{*}$ \\
\hline & Sham rTMS & $35.5 \pm 7.1$ & $35.6 \pm 7.2$ & $35.4 \pm 7.0$ & 0.943 & 0.957 \\
\hline
\end{tabular}

Values are presented as mean \pm standard deviation.

SOT, sensory organization test; DCL, directional control; BBS, Berg Balance Scale; rTMS, repetitive transcranial magnetic stimulation.

${ }^{*} \mathrm{p}<0.05$. 
(A)

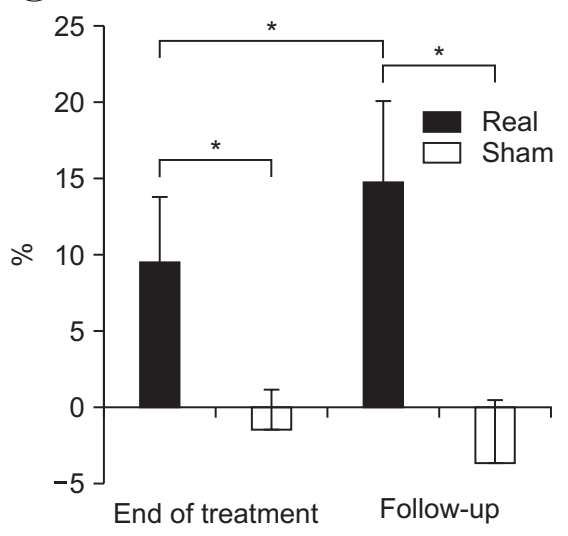

(D)

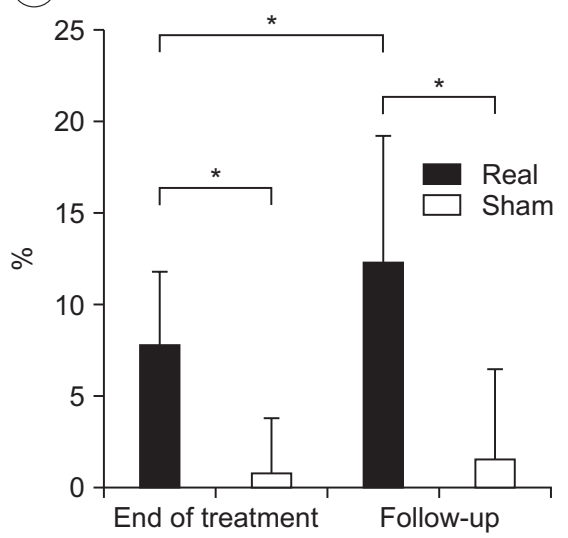

(B)

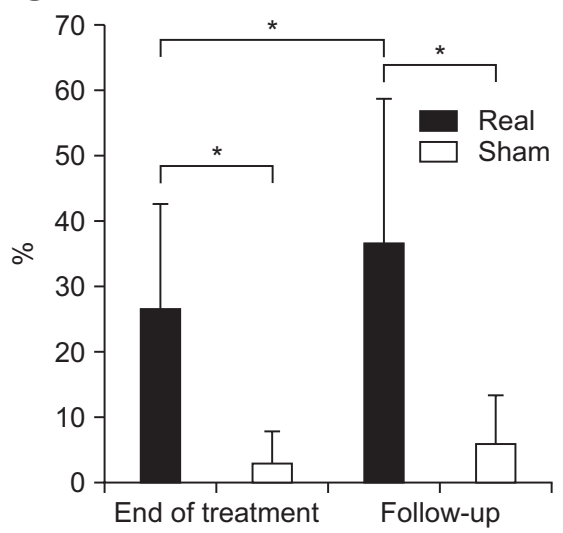

(E)

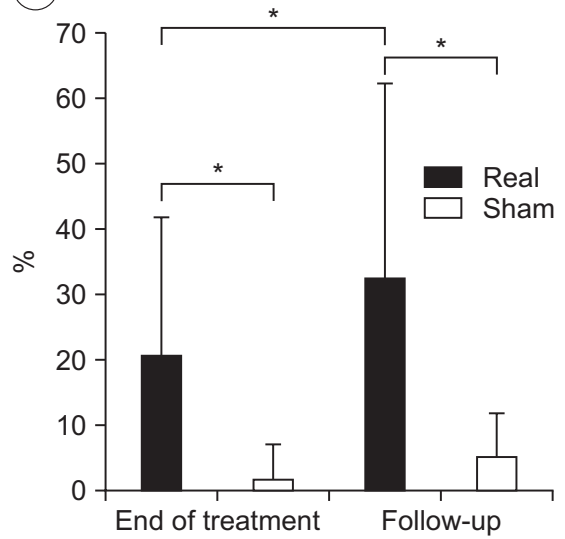

(C)

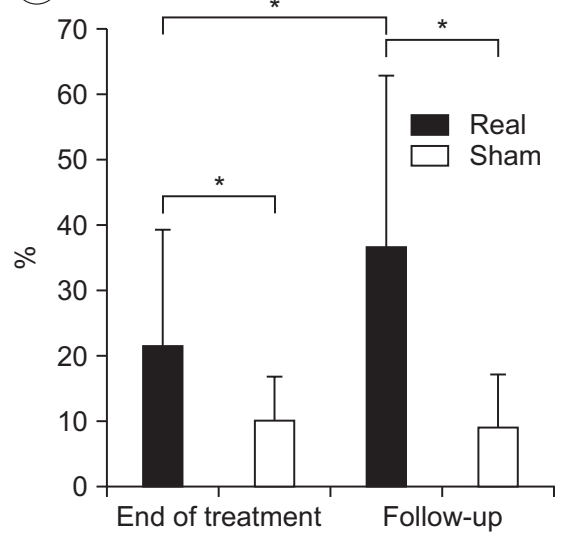

Fig. 3. The percentage improvement in clinical measures. (A) SOT, significant improvement at the end of treatment as well as at follow-up. Other clinical measures also showed similar results. (B) On-axis velocity left-right, (C) on-axis velocity front-back, (D) DCL left-right, and (E) DCL front-back. SOT, sensory organization test; DCL, directional control. ${ }^{*} \mathrm{p}<0.05$.

balance function in the cerebral infarction patients. Few other studies have demonstrated that control of trunk muscles is associated with balance function and activities of daily living [19-21]. It means that the restoration of trunk muscle positively influences the balance function in the patients with cerebral infarction. Park et al. [22] reported that the expression patterns of evoked potential of trunk muscles in patients with unilateral hemispheric stroke are different from that of normal people, and is accompanied with damage of motor nerve pathway of trunk muscles after stroke. Therefore, we assumed that rTMS could induce positive effect on the balance function when rTMS is applied to the trunk area of the primary cortex as it influences damaged motor nerve circuit of trunk muscles.

In the study involving normal adults, activity of thoracic erector spinae muscles increased when they maintain balance in unstable physical posture [23]. In a study carried out by Dickstein et al. [24], which compared trunk muscle strength between normal people and hemiplegic stroke patients using surface electrode, hemiplegic stroke patients showed significantly weak activity in erector spinae muscles but not in bilateral rectus abdominis, external abdominis oblique, and latissimus dorsi muscles. Another study showed delay in latency of erector spinae muscles in hemiplegic stroke patients as compared with normal people when arms were bent in sitting position [25]. Also, the study which investigated probability of occurrence of MEP in the contralateral side when TMS was applied to the affected cerebral hemisphere in stroke patients, revealed that MEP of 9th thoracic erector spinae muscles occurred in $\mathbf{8 0 \%}$ of subjects and the frequency 
was same or of higher level when compared with that of other trunk muscles [22]. Based on the reported studies, we applied rTMS on the cortical motor area of 9th thoracic erecter spinae muscles.

To the best of our knowledge, until date there exists no study about applying therapeutic rTMS over the trunk motor spot of the stroke patient with balance deficits. There was a report stating improvement in balance and lower limb strength upon application of tDCS to the patients with hemiplegic stroke [15], and another study reported improvement in lower limb function when high frequency rTMS using H-coil was applied to the chronic stroke patients with effect lasting for more than a month [26].

This study suggests that the therapeutic effect of rTMS treatment in patients lasts not only right after the treatment, but also after a month from the initiation of study. However, our results showed a tendency to decline for SOT of T4 and T5 for real-sham rTMS group, although the result of the 2 time points (T4, T5) significantly improved as compared with that of baseline (T1) (Fig. 2). We believe that the effect of rTMS on balance function might fade away with time after 1 month. Further studies on effect of duration and mechanism of rTMS are necessitated.

Application of rTMS over the motor cortex is based on the interhemispheric competition model after cerebral infarction $[10,27,28]$. Subcortical stroke studies reveal that imbalance of interhemispheric inhibition occurs in chronic stroke patients $[27,29]$. After the occurrence of cerebral infarction, the intact motor cortex was disinhibited by decreased transcallosal inhibition from the affected motor cortex. Therefore, decreased excitability was observed in affected motor cortex as compared with intact motor cortex in chronic hemiparetic patients [30]. Previous studies consider that these abnormal interhemispheric inhibitions impede functional motor recovery in stroke patients [27] and motor function can be restored by decreasing transcallosal inhibition from intact motor $[10,31]$. Avenanti et al. [32] reported that the use-dependent motor plasticity can be promoted and motor excitability of interhemisphere can be readjusted when rTMS is applied to chronic stroke patients. In this context, our results support the results from the previous reported studies.

This study has some limitations. First, because the lower extremity motor cortex is close to the trunk motor cor- tex, rTMS might affect not only trunk but also lower extremity. Therefore, there is a possibility that the balance function would be improved by effect of rTMS on lower extremity motor cortex. However, manual muscle test (MMT) grades of lower extremity did not show significant change between before and after treatments. Therefore, it was hard to believe that lower extremity motor cortex was affected in this study. Second, the sample size was small (i.e., $\mathrm{n}=30$ ). Third, there was a limitation for measuring the precise effect of the real rTMS since both the washout period and the follow-up period were short. Thus, future studies involving longer follow-up and wash-out period are required. Even if the present study had a relatively short wash-out period of four weeks, it was aimed to reduce errors by performing both the real rTMS and sham rTMS in all patients through the cross-over study. Fourth, this study covered patients with mild to moderate balance impairment. Nevertheless, we could not evaluate the balance function in patients with severe balance deficits using computerized dynamic posturography. Therefore, another evaluation method is required to evaluate the balance function.

In conclusion, to the best of our knowledge, this is the first study to investigate the therapeutic effects of rTMS over the trunk motor cortex on balance function in stroke patients. Our results show that high frequency rTMS may be used as one of the strategies for treating chronic stroke patients with balance impairment. Further studies are required to assess not only underlying mechanisms but also detailed protocol.

\section{CONFLICT OF INTEREST}

No potential conflict of interest relevant to this article was reported.

\section{REFERENCES}

1. Alberts JL, Wolf SL. The use of kinetics as a marker for manual dexterity after stroke and stroke recovery. Top Stroke Rehabil 2009;16:223-36.

2. Scott WG, Scott H. Ischaemic stroke in New Zealand: an economic study. N Z Med J 1994;107:443-6.

3. Tyson SF, Hanley M, Chillala J, Selley AB, Tallis RC. The relationship between balance, disability, and recovery after stroke: predictive validity of the Brunel 
Balance Assessment. Neurorehabil Neural Repair 2007;21:341-6.

4. Laufer Y, Sivan D, Schwarzmann R, Sprecher E. Standing balance and functional recovery of patients with right and left hemiparesis in the early stages of rehabilitation. Neurorehabil Neural Repair 2003;17:20713.

5. Geiger RA, Allen JB, O'Keefe J, Hicks RR. Balance and mobility following stroke: effects of physical therapy interventions with and without biofeedback/forceplate training. Phys Ther 2001;81:995-1005.

6. Bourbonnais D, Bilodeau S, Lepage Y, Beaudoin N, Gravel D, Forget R. Effect of force-feedback treatments in patients with chronic motor deficits after a stroke. Am J Phys Med Rehabil 2002;81:890-7.

7. Juneja G, Czyrny JJ, Linn RT. Admission balance and outcomes of patients admitted for acute inpatient rehabilitation. Am J Phys Med Rehabil 1998;77:388-93.

8. Nichols DS, Miller L, Colby LA, Pease WS. Sitting balance: its relation to function in individuals with hemiparesis. Arch Phys Med Rehabil 1996;77:865-9.

9. Rubenstein LZ, Josephson KR. The epidemiology of falls and syncope. Clin Geriatr Med 2002;18:141-58.

10. Nowak DA, Grefkes C, Ameli M, Fink GR. Interhemispheric competition after stroke: brain stimulation to enhance recovery of function of the affected hand. Neurorehabil Neural Repair 2009;23:641-56.

11. Webster BR, Celnik PA, Cohen LG. Noninvasive brain stimulation in stroke rehabilitation. NeuroRx 2006;3:474-81.

12. Fregni F, Boggio PS, Mansur CG, Wagner T, Ferreira MJ, Lima MC, et al. Transcranial direct current stimulation of the unaffected hemisphere in stroke patients. Neuroreport 2005;16:1551-5.

13. Hummel FC, Cohen LG. Non-invasive brain stimulation: a new strategy to improve neurorehabilitation after stroke? Lancet Neurol 2006;5:708-12.

14. Lefaucheur JP, Andre-Obadia N, Antal A, Ayache SS, Baeken C, Benninger DH, et al. Evidence-based guidelines on the therapeutic use of repetitive transcranial magnetic stimulation (rTMS). Clin Neurophysiol 2014;125:2150-206.

15. Sohn MK, Jee SJ, Kim YW. Effect of transcranial direct current stimulation on postural stability and lower extremity strength in hemiplegic stroke patients. Ann Rehabil Med 2013;37:759-65.
16. Franchignoni FP, Tesio L, Ricupero C, Martino MT. Trunk control test as an early predictor of stroke rehabilitation outcome. Stroke 1997;28:1382-5.

17. Hsieh CL, Sheu CF, Hsueh IP, Wang CH. Trunk control as an early predictor of comprehensive activities of daily living function in stroke patients. Stroke 2002;33:2626-30.

18. Karatas M, Cetin N, Bayramoglu M, Dilek A. Trunk muscle strength in relation to balance and functional disability in unihemispheric stroke patients. Am J Phys Med Rehabil 2004;83:81-7.

19. Verheyden G, Vereeck L, Truijen S, Troch M, Herregodts I, Lafosse C, et al. Trunk performance after stroke and the relationship with balance, gait and functional ability. Clin Rehabil 2006;20:451-8.

20. Likhi M, Jidesh VV, Kanagaraj R, George JK. Does trunk, arm, or leg control correlate best with overall function in stroke subjects? Top Stroke Rehabil 2013;20:62-7.

21. Kobayashi M, Takahashi K, Sato M, Usuda S. Association of performance of standing turns with physical impairments and walking ability in patients with hemiparetic stroke. J Phys Ther Sci 2015;27:75-8.

22. Park SH, Song KS, Ko MH, Seo JH. Motor evoked potentials of trunk muscles in stroke patients. J Korean Acad Rehabil Med 2009;33:282-9.

23. O'Sullivan PB, Grahamslaw KM, Kendell M, Lapenskie SC, Moller NE, Richards KV. The effect of different standing and sitting postures on trunk muscle activity in a pain-free population. Spine (Phila Pa 1976) 2002;27:1238-44.

24. Dickstein R, Sheffi S, Ben Haim Z, Shabtai E, Markovici E. Activation of flexor and extensor trunk muscles in hemiparesis. Am J Phys Med Rehabil 2000;79:22834.

25. Dickstein R, Shefi S, Marcovitz E, Villa Y. Anticipatory postural adjustment in selected trunk muscles in post stroke hemiparetic patients. Arch Phys Med Rehabil 2004;85:261-7.

26. Chieffo R, De Prezzo S, Houdayer E, Nuara A, Di Maggio G, Coppi E, et al. Deep repetitive transcranial magnetic stimulation with $\mathrm{H}$-coil on lower limb motor function in chronic stroke: a pilot study. Arch Phys Med Rehabil 2014;95:1141-7.

27. Murase N, Duque J, Mazzocchio R, Cohen LG. Influence of interhemispheric interactions on motor func- 
tion in chronic stroke. Ann Neurol 2004;55:400-9.

28. Shimizu T, Hosaki A, Hino T, Sato M, Komori T, Hirai $\mathrm{S}$, et al. Motor cortical disinhibition in the unaffected hemisphere after unilateral cortical stroke. Brain 2002 Aug;125(Pt 8):1896-907.

29. Liepert J, Hamzei F, Weiller C. Motor cortex disinhibition of the unaffected hemisphere after acute stroke. Muscle Nerve 2000;23:1761-3.

30. Liepert J, Restemeyer C, Kucinski T, Zittel S, Weiller C. Motor strokes: the lesion location determines motor excitability changes. Stroke 2005;36:2648-53.

31. Takeuchi N, Chuma T, Matsuo Y, Watanabe I, Ikoma K. Repetitive transcranial magnetic stimulation of contralesional primary motor cortex improves hand function after stroke. Stroke 2005;36:2681-6.

32. Avenanti A, Coccia M, Ladavas E, Provinciali L, Ceravolo MG. Low-frequency rTMS promotes use-dependent motor plasticity in chronic stroke: a randomized trial. Neurology 2012;78:256-64. 